\title{
CELL SCHEDULING AND BANDWIDTH ALLOCATION FOR HETEROGENEOUS VBR VIDEO CONFERENCING TRAFFIC
}

\author{
Tao Yang*, Danny H.K. Tsang $\dagger$, and Patrick McCabe* \\ *Department of Industrial Engineering, Technical University of Nova Scotia \\ Halifax, Nova Scotia, Canada B3J 2X4 \\ yangt@newton.tuns.ca \\ $\dagger$ Department of Electrical and Electronic Eng., Hong Kong University of Science and Technology \\ Clear Water Bay, Kowloon, Hong Kong \\ eetsang@uxmail.ust.hk
}

\begin{abstract}
We first propose a new cell loss scheduling scheme, referred to as the $Q o S$-scheme. This scheme can satisfy different individual quality of service $(\mathrm{QoS})$ requirements of a group of virtual channel connections with minimum required bandwidth. We next derive a simple formula based on Gaussian approximation to allocate bandwidth to heterogeneous VBR video conferencing connections. Comparing the proposed QoS-scheme with the simple last-in-first-out (LIFO) scheme, we find that if the LIFO scheme is adopted as the loss scheduling scheme, developing any more efficient bandwidth allocation algorithms can at most improve bandwidth utilization by about $10 \%$. However, if the proposed QoS-scheme is implemented, an efficient allocation algorithm can improve bandwidth utilization by roughly $20 \%$.
\end{abstract}

\section{Introduction}

Real-time video (e.g., video conferencing, video-on-demand, and switched broadcasted TV programs) is expected to be one of the major services supported by ATM-based B-ISDN. Real-time video traffic can be transmitted in variable-bit-rate (VBR) or constant-bit-rate (CBR). VBR video can take the advantage of statistical multiplexing gain offered by the ATM technology and, therefore, is potentially more efficient than the CBR counterpart in bandwidth utilization. However, network resource management of VBR video connections is far more complicated than that of CBR connections. In this paper, we investigate various issues concerning resource management for VBR video conferencing connections in the ATM network. The objective is to determine an effective cell loss scheduling scheme coupled with a simple and relatively accurate bandwidth allocation algorithm for VBR video conferencing traffic.

More specifically, we consider the traffic model described in Fig. 1. Here, a number of virtual channel connections (VCC), each carrying VBR video conferencing traffic from a particular source, are multiplexed into a common virtual path connection (VPC) by an ATM multiplexer or switch. All VCC share the bandwidth allocated to the VPC and they may have different traffic characteristics and different quality of service (QoS) requirements. We are interested in determining an efficient and effective cell loss scheduling scheme and a simple bandwidth allocation algorithm with which we can satisfy individual QoS requirements of all VCC involved and, at the same time, achieve high bandwidth utilization.
Bandwidth allocation has been the focus of intense research in recent years and numerous approaches have been proposed from both industry and academia (see, e.g., [1, 3, 6, 8, 10-12, 14, $15,17,18,19])$. Most of these works were for VBR connections of on-off sources and only a few were for VBR video traffic. Due to complexity of VBR traffic, especially those of VBR video connections, an accurate estimation usually requires a considerable amount of computation time, making it impossible for realtime implementation. Hence, the strategy is to develop a simple and accurate estimation method. A number of outstanding techniques have been developed for on-and-off VBR traffic. Among them are two most computationally efficient approaches, namely, the effective bandwidth approach $[4,5,7,13]$ and the stationary Gaussian approximation [7]. In [2, 21], these two approaches have been extended to accommodate VBR video conferencing connections with the same QoS requirement. It was found that the effective bandwidth approach over-estimates the bandwidth by a large quantity; that the Gaussian approximation slightly over-estimates the bandwidth and is much more accurate than when it is applied to VBR traffic of on-and-off sources.

In this paper, we further extend the Gaussian approximation approach to cases where all VCC are allowed to have different QoS requirements. We also conduct detailed simulation studies on the traffic model in Fig. 1 to examine the accuracy and applicability of the Gaussian approximation when it is applied to VBR video conferencing traffic.

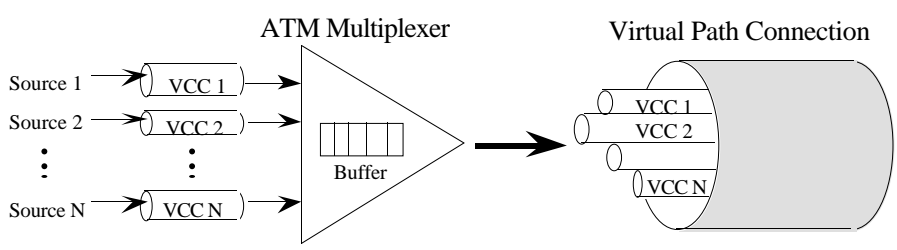

Fig. 1. The traffic model of an ATM node

The second major issue concerns cell loss scheduling or cell discarding schemes. The simplest is the last-in-first-out (LIFO) scheme in which cells are transmitted through the VPC in the order of arrival regardless of their VCC identity; however, when a cell arrives and the buffer is full, it is discarded, again, regardless of its VCC identity. The major advantage of this scheme is its 
implementation simplicity. However, it raises the problems of traffic interference and the waste of bandwidth due to multiple QoS requirements. Traffic interference among different VCC causes individual connections to experience different cell loss ratios (see, e.g., $[9,16,17,20]$ ). If bandwidth allocation is based on the measure of overall cell loss ratio, then the cell loss ratio requirement of some connections may not be satisfied even when the overall cell loss ratio is satisfied. One solution to this problem is to allocate bandwidth based on individual performance measures (see, e.g., $[16,17])$ and this requires sophisticated computation to estimate the required bandwidth. Another solution, initially proposed in [9] for homogeneous VCC and later extended in [21] to heterogeneous VCC, is to eliminate the effect of traffic interference by proper cell loss scheduling.

Although a proper cell loss scheduling scheme can ensure that all VCC experience almost the same cell loss ratio, the problem of waste of bandwidth still remains if the VCC have different QoS requirements (or more specifically, different cell loss requirements). In this case, a common practice is to apply the most stringent QoS requirement to all VCC and their bandwidth requirement is calculated accordingly (see, e.g., [7]). This however may introduce reduction in bandwidth utilization since some connections may experience a better cell loss ratio than they asked for. At this point, one might ask: Is it possible to satisfy individual QoS requirements without bandwidth waste using an ideal cell scheduling scheme? If yes, how? How significant is the waste of bandwidth caused by the simple LIFO scheme when compared with the ideal scheduling scheme? Finally, is it worth implementing such an ideal yet most likely complicated scheduling scheme?

To answer the above questions, we have developed a new cell loss scheduling scheme, referred to as the QoS-scheme, which not only eliminates the effect of traffic interference but also ensures that the individual cell loss requirement of any VCC can be just satisfied. Since the cell loss requirement is not over-satisfied, this cell loss scheduling scheme yields the best (or the optimal) bandwidth utilization. Although this ideal scheduling scheme may be complicated to implement in an ATM switch, we use it as a reference to compare the performance of various existing schemes, in particular the LIFO scheme; moreover, for the bandwidth allocation problem, it is used to determine a suitable tradeoff between bandwidth utilization and algorithm simplicity.

Due to the complexity of VBR video conferencing traffic coupled with the cell scheduling scheme, an accurate queueing analysis is mathematically intractable. Therefore, we base our study on detailed simulations with the VBR video traffic generated from a long sequence of real video conferencing data (over 38,000 frames or 21 minutes). Our results suggest that although traffic interference in the case of the simple LIFO scheme has a significant impact on cell loss performance experienced by individual connections, it has a minor effect on the extra amount of bandwidth required by all the connections that are multiplexed into a common virtual path. As far as cell scheduling schemes are concerned, our simulations show that the simple LIFO scheme requires about 10 percent more bandwidth than the ideal QoS-scheme. For bandwidth allocation, we find that the stationary Gaussian approximation is generally more accurate than when it is applied to VBR traffic of the on-and-off type [7]; and that the stationary Gaussian approximation consistently over-estimates the required bandwidth by about 10 percent compared with the simulation results. This over-estimation of bandwidth provides the safety margin to reduce the possibility of temporally QoS violations caused by traffic interference.

Our findings presented in this paper seem to suggest that VCC carrying video conferencing traffic should be multiplexed into a single VPC with dedicated bandwidth and a LIFO buffer. A simple formula based on stationary Gaussian approximation can be used to calculate the amount of required bandwidth using the most stringent QoS requirement. This setup has several attractive features. First, it is easy to implement and does not require sophisticated buffer management and cell scheduling schemes. Second, the impact of traffic interference among connections having different traffic characteristics is alleviated by providing sufficient bandwidth. Third, individual QoS requirements are satisfied. Finally, it has relatively high bandwidth utilization.

The rest of the paper is organized as follows. In Section 2, the system model and VBR video traffic are described in more detail. In Section 3, the QoS cell scheduling scheme (QoSscheme) is introduced and its performance is compared using simulation with those of other scheduling schemes proposed in the literature. In Section 4, we present a description of the stationary Gaussian approximation and how it can be applied to estimate bandwidth needed by a group of VCC to satisfy their QoS requirements. Two approximation methods are proposed and their accuracy is examined against simulation results of the traffic model under different cell scheduling schemes. Finally, in Section 5, conclusions are drawn from the findings and recommendations are made for transporting VBR video conferencing traffic in ATM networks.

\section{The Traffic Model}

Let us consider the traffic model as shown in Fig. 1. In particular, we assume that the ATM multiplexer (or switch) has a buffer capacity of $K$ cells dedicated to the virtual path connection (VPC) with a link capacity of $C$ bits per second (bps). Cells from the $N$ VCC are transmitted through the VPC on first-infirst-out basis. When a cell arrives and the buffer is full, either the arriving cell or one of the cells already in the buffer will be discarded according to a certain cell loss scheduling scheme. The scheduling scheme that always discard the arriving cell is called the LIFO-scheme. Other cell loss scheduling schemes are discussed in the next section.

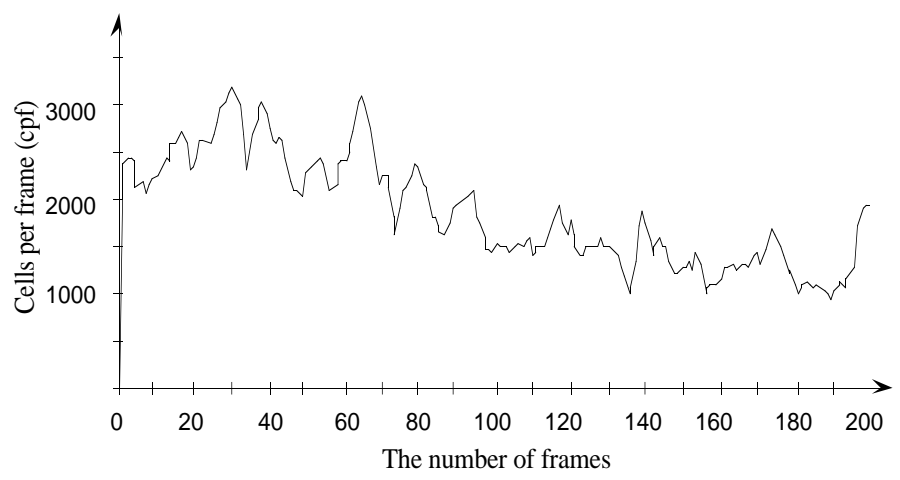

Fig. 2. A sample path of $\left\{X_{n}^{(i)}, n \geq 0\right\}$

Each VCC carries the traffic from a VBR video conferencing source that generates 30 frames per second. Due to video compression, the amount of information contained in a frame, 
measured in cells per frame (cpf) or bits per second (bps), varies over time stochastically. Let us define $X_{n}^{(i)}$ as the number of cells in the $n$th frame from the $i$ th video source and use the sequence $\left\{X_{n}^{(i)}, n \geq 0\right\}$ to represent the traffic generated by the $i$ th video source. Fig. 2 shows a sample path of a video sequence generated by a DPCM/DCT codec. The cells within a frame are transmitted at a constant rate during the frame interval $(1 / 30 \mathrm{sec}-$ onds). Hence, the source cell rate may change only at frame boundaries.

A VBR video sequence has two important properties that may not hold for other video sequences such as VBR MPEG video traffic. The first is the stationary property that states that the sequence $\left\{X_{n}^{(i)}, n \geq 0\right\}$ behaves more or less stationary over time [9]. The second property is that a video conferencing sequence does not have frequent scene changes. In other words, abrupt changes in the sequence $\left\{X_{n}^{(i)}, n \geq 0\right\}$ are unlikely to be observed. Since $\left\{X_{n}^{(i)}, n \geq 0\right\}$ is stationary, we can define some of its limiting statistics. In this paper two important statistics, the mean and the variance of $X_{n}^{(i)}$, will be used. They are defined as follows

$$
\mu_{i} \equiv E\left[X_{n}^{(i)}\right] \text { and } \sigma_{i}^{2} \equiv \operatorname{var}\left[X_{n}^{(i)}\right]
$$

where the use of subscripts implies that different sources (or connections) may have different statistical characteristics. We point out that other characteristics such as the peak rate $\sup _{n \geq 0}\left\{X_{n}^{(i)}\right\}$ and those characterizing the correlation are also important for other purposes (e.g., the peak rate can be used for policing the incoming traffic). Finally, we assume that the VCC may have different QoS requirements. Among all the potential QoS parameters, we are only interested in the cell loss ratio and use $C L R_{i}$ to denote the cell loss ratio specification for the $i$ th source (or VCC $i$ ). Cell delay and cell delay variation can be accommodated using a relatively small buffer.

The aggregate traffic of several VCC carrying VBR video conferencing traffic is far more complicated. Since the frames from different connections are not synchronized, the relative positions of the frame boundaries of the VCC are randomly distributed but this random pattern occurs periodically (i.e., every $1 / 30$ second). Performing a complete queueing analysis to obtain performance measures concerning the various cell loss scheduling schemes would be mathematically intractable. Our traffic model is therefore represented by an event driven simulation using data generated from the real VBR video conferencing sequence. The event set of our discrete event system are $N$ cell arrivals (one from each of the $N$ sources) and one departure.

Table 1. Traffic characteristics and cell loss requirements $(\mathrm{cpf}=$ cells per frame; $1 \mathrm{cpf}=12,720 \mathrm{bps})$

\begin{tabular}{|c||c|c|c|}
\hline & Class 1 & Class 2 & Class 3 \\
\hline \hline$\mu$ & $75.3 \mathrm{cpf}$ & $301.3 \mathrm{cpf}$ & $150.7 \mathrm{cpf}$ \\
\hline$\sigma$ & $25.6 \mathrm{cpf}$ & $102.5 \mathrm{cpf}$ & $51.3 \mathrm{cpf}$ \\
\hline$C L R$ & $10^{-3}$ & $10^{-4}$ & $10^{-5}$ \\
\hline
\end{tabular}

Since the sequence $\left\{X_{n}^{(i)}, n \geq 0\right\}$ is a stationary stochastic process we can generate different video sequences (having the same statistical characteristics) from one data sequence using the method suggested in [9]. This is done by simply using the existing data sequence, starting at a randomly selected initial frame, and proceeding through the data sequence while considering it as a circular list. In this paper, we generate all of our source traffic from a sequence of real VBR video conferencing data (over 38,000 frames) obtained from a DPCM/DCT codec.

To speed up the simulation whose running time depends on the total number of cells processed, we scale the real data by a factor of $\beta$, where $0<\beta<1$. For example, if $\left\{X_{n}, n \geq 0\right\}$ is the real data sequence, the scaled sequence is $\left\{\beta X_{n}, n \geq 0\right\}$ that has a smaller number of cells per frame. To generate sequences with different statistical characteristics, we apply different scaling factors to the real data sequence. Three classes of source data are produced by scaling down the original sequence by factors of $0.05,0.2$, and 0.1 , respectively. These are referred to as Class 1 , Class 2, and Class 3, respectively; their corresponding traffic statistics as well as the cell loss ratio (CLR) requirements are given in Table 1.

\section{Cell Loss Scheduling Based on QoS Measurements}

Cell loss scheduling is a technique used to determine which cell to discard especially when the buffer is full. The simplest scheme, called the LIFO-scheme, is to discard the arriving cell whenever the buffer is full. Under this scheme, different VCC may experience different cell loss ratios even when they have the same traffic characteristics and the same cell loss requirements. To overcome this problem, a cell loss scheduling scheme was proposed in [9]; its basic idea is described as follows. For each VCC, say VCC $i$, a loss counter $L_{i}$ is used to record the total number of discarded cells up to the present time. When a cell from VCC $i$ arrives and the buffer is full, the VCC that has the smallest counter value and has at least one cell in the buffer is selected. Suppose it is VCC $j$. If $i \neq j$ and $L_{i}>L_{j}$, then the last cell from VCC $j$ in the buffer is discarded, $L_{j}$ is increased by one, and the arriving cell joins the end of the queue. Otherwise, the arriving cell is discarded and $L_{i}$ is increased by one. This cell loss scheduling scheme makes an effort to ensure that the total number of discarded cells from each VCC is equal. This scheduling scheme is referred to as the L-scheme.

Since the L-scheme tends to even out the total numbers of discarded cells (not the cell loss ratio) among all VCC, individual cell loss ratios can still be different if the VCC do not have the same traffic characteristics or if they have different connection (session) intervals (e.g., one VCC's session time can be 10 minutes while the other is only 2 minutes). In the heterogeneous traffic case, VCC with heavy traffic usually experience smaller cell loss ratios than those with light traffic. In the case of different session intervals, VCC that have shorter session intervals and have started later than those with longer session intervals may experience larger cell loss ratios.

To overcome this difficulty, the L-scheme is modified in [21]. Here, in addition to the counter $L_{i}$, another counter, called $A_{i}$, is used to record the total number of cells having arrived from VCC $i$; the cell loss ratio $R_{i}=L_{i} / A_{i}$ is calculated each time the arriving cell sees the buffer full. When a cell from VCC $i$ arrives and the buffer is full, the VCC that has the smallest cell loss ratio and has at least one cell in the buffer is determined. Suppose it is VCC $j$. If $i \neq j$ and $R_{i}>R_{j}$, then the last cell from VCC $j$ in the buffer is discarded, $L_{j}$ and $R_{j}$ are updated, and the arriving cell joins the end of the queue. Otherwise, the arriving cell is discarded and $L_{i}, A_{i}$, and $R_{i}$ are updated. This scheduling scheme attempts to even out the cell loss ratios among VCC with different traffic characteristics and session intervals. This is referred to as the $R$-scheme. 
The R-scheme is applicable when all VCC have the same cell loss ratio requirements. If several VCC have different QoS loss requirements, then it makes no sense to schedule their cells to even out their cell loss ratios. We thus introduce a new scheduling scheme called the $Q o S$-scheme which attempts to force the cell loss ratio of each VCC to approach as close as possible towards its targeted cell loss ratio (CLR) specification given in the traffic contract. This scheme calculates the ratio $R_{i}$ as defined in the R-scheme and takes it a step further by calculating the relative error with respect to the target value $C L R_{i}$ :

$$
E_{i}=\left(R_{i}-C L R_{i}\right) / C L R_{i} .
$$

When a cell from VCC $i$ arrives and the buffer is full, the VCC that has the smallest relative error and has at least one cell in the buffer is selected. Again, suppose it is VCC $j$. If $i \neq j$ and $E_{i}$ $>E_{j}$, then the last cell from VCC $j$ in the buffer is discarded, $L_{j}, R_{j}$, and $E_{j}$ are updated, and the arriving cell joins the end of the queue. Otherwise, the arriving cell is discarded, and $L_{i}, A_{i}$, $R_{i}$, and $E_{i}$ are updated. In theory, this cell loss scheduling scheme can meet the individual cell loss ratio of a VCC with minimum bandwidth requirement.

To compare the performance of the above four cell scheduling schemes, we have done extensive simulation studies. Here, we present some of the results. The first set of simulations were run with 10 homogeneous sources that are drawn from Class 1 as defined in Table 1 . We use a bandwidth of $C=$ $11.909 \mathrm{Mbps}$ and a buffer size of $K=100$ cells. The results are shown in Table 2 as follows.

Table 2. Sample measures of cell loss ratio $\left(\times 10^{-3}\right)$ for homogeneous sources with the same $C L R$ requirement

\begin{tabular}{|c||c|c|c|c|}
\hline \multicolumn{1}{|c||}{ Source } & \multicolumn{4}{c|}{ Loss scheduling scheme } \\
\cline { 2 - 5 } & LIFO & L-sch. & R-sch. & QoS-sch. \\
\hline \hline 1 & 1.04 & 1.00 & 1.00 & 1.00 \\
\hline 2 & 0.84 & 1.00 & 1.00 & 1.00 \\
\hline 3 & 1.07 & 1.00 & 1.00 & 1.00 \\
\hline 4 & 0.99 & 1.00 & 1.00 & 1.00 \\
\hline 5 & 0.92 & 1.00 & 0.99 & 0.99 \\
\hline 6 & 0.96 & 1.00 & 1.00 & 1.00 \\
\hline 7 & 1.08 & 1.00 & 1.00 & 1.00 \\
\hline 8 & 0.95 & 1.00 & 0.99 & 0.99 \\
\hline 9 & 1.05 & 1.00 & 1.00 & 1.00 \\
\hline 10 & 1.08 & 1.00 & 0.99 & 0.99 \\
\hline
\end{tabular}

For the LIFO-scheme the sample cell loss ratios have the largest variation as expected. The L-scheme forces the cell loss ratio of each VCC to exactly the same value. For homogeneous sources generated from the same sequence of data (see discussions in the last section) the total number of arriving cells are the same. Coupled with the fact that the L-scheme matches the number of cell losses, it explains why L-scheme gives identical cell loss ratios for each homogeneous source. The R-scheme evens out the cell losses more than LIFO but not as much as the L-scheme does. The cell loss ratio $R_{i}$ is dynamically changing as cells arrive so the cell discarding policy in the R-scheme as compared to the L-scheme is different: some VCC may have more cells arrived before those of other VCC but the decision to discard cells in the R-scheme does not anticipate the fact that in the end of the simulation the numbers of arrivals for all VCC will be the same. Since all sources have the same $C L R$ requirements, the QoS-scheme acts identical to the R-scheme. Our conclusion is that for homogeneous sources with the same CLR value, the LIFO-scheme performs the worst; the L-scheme performs the best; and the R-scheme and the QoS-scheme are almost as good as the L-scheme.

What happens when we need to multiplex VCC with different traffic characteristics and different cell loss ratio requirements? To see this, we present the next set of simulations which uses 4 VCC from each of the 3 classes defined in Table 1 . We use a bandwidth of $C=35.535 \mathrm{Mbps}$ and a buffer size of $K=50$ cells. Table 3 shows the cell loss ratios from this set of simulations. The L-scheme matches cell losses among all VCC: within each class, the L-scheme leads to similar cell loss ratios as already seen in Table 2. Since each of the 3 classes has a drastically different number of cells that arrive, the L-scheme leads to very different cell loss ratios across the different classes. For example, sources from Class 3 that have the most strict QoS requirement end up having a cell loss ratio larger than those sources of Class 2 that have a less strict QoS requirement. The Rscheme leads to cell loss ratios quite similar across all sources irrespective of class. This scheme performs well if all sources had the same QoS requirements, but unfortunately with multiple QoS requirements the R-scheme falls short.

Table 3. Sample measures of cell loss ratios $\left(\times 10^{-4}\right)$ for heterogeneous sources

\begin{tabular}{|c|c||c|c|c|c|}
\hline \multicolumn{2}{|c||}{$\begin{array}{c}\text { Source } \\
(C L R)\end{array}$} & \multicolumn{4}{c|}{ Loss scheduling scheme } \\
\cline { 2 - 6 } & 1 & 1.869 & 4.670 & 1.998 & 9.866 \\
\cline { 2 - 6 } Class 1 & 2 & 1.782 & 4.670 & 1.970 & 9.734 \\
\cline { 2 - 6 }$\left(10^{-3}\right)$ & 3 & 1.810 & 4.670 & 2.005 & 9.911 \\
\cline { 2 - 6 } & 4 & 2.474 & 4.670 & 1.994 & 9.862 \\
\hline \multirow{4}{*}{$\begin{array}{c}\text { Class } 2 \\
\left(10^{-4}\right)\end{array}$} & 5 & 1.652 & 1.168 & 2.015 & 0.996 \\
\cline { 2 - 6 } & 7 & 2.333 & 1.168 & 2.024 & 1.000 \\
\cline { 2 - 6 } & 8 & 1.961 & 1.167 & 1.995 & 0.986 \\
\hline \multirow{4}{*}{$\begin{array}{c}\text { Class 3 } \\
\left(10^{-5}\right)\end{array}$} & 9 & 2.012 & 2.333 & 2.017 & 0.099 \\
\cline { 2 - 6 } & 10 & 1.996 & 2.333 & 1.987 & 0.097 \\
\cline { 2 - 6 } & 12 & 1.751 & 2.333 & 2.001 & 0.097 \\
\hline
\end{tabular}

However, the QoS-scheme deals well with multiple QoS requirements. Instead of evening out cell losses or cell loss ratios, the QoS-scheme evens out the relative errors that occur from the differences between the actual cell loss ratios and the corresponding CLR requirements. We have observed the following in our simulation experiments: for small output bandwidth such that all CLR requirements are violated under all schemes, the QoSscheme will try to even out the degree of violation across all classes and sources; for large output bandwidth such that the CLR requirements are exceeded under all four schemes, the QoSscheme will try to even out the degree of surplus across all classes and sources. Thus, we conclude that QoS-scheme is a fair loss scheduling discipline. 


\section{Bandwidth Allocation}

The key issue in bandwidth allocation is to calculate the amount of bandwidth required by a group of VCC given their traffic characteristics and QoS requirements. The tradeoff in a bandwidth allocation algorithm is between its simplicity and accuracy. A simple formula for bandwidth allocation may be used in real-time connection admission control (CAC) to decide whether the available bandwidth can accommodate a new connection, while an accurate formula usually results in higher bandwidth utilization. As mentioned in the first section, two simple but reasonably accurate methods, namely the effective bandwidth approach and the stationary Gaussian approximation, have been proposed for VBR connections of on-and-off sources. In $[2,21]$, these two methods have been adapted to VBR video conferencing traffic with the same QoS requirement. The conclusions from these studies suggest that stationary Gaussian approximation performs well while the effective bandwidth approach over-estimates the bandwidth by a large quantity. Therefore, we only consider the former approach in the rest of this paper.

The objective of this section is three-fold. First, we will extend the Gaussian approximation to the case of VBR video conferencing connections with different cell loss ratio requirements. Next, we will determine which of the four cell loss scheduling schemes can be coupled with the Gaussian approximation, i.e., we will examine whether the amount of bandwidth calculated using the Gaussian approximation is sufficient to satisfy the cell loss ratio requirements of all VCC under each of the four scheduling schemes. Finally, we hope to determine the best cell scheduling scheme regarding simplicity, bandwidth utilization, and individual QoS guarantees.

According to the Gaussian approximation, for a VBR traffic stream whose rate has a mean of $\mu$ and a standard deviation of $\sigma$, the required bandwidth is estimated to be [7]:

$$
\hat{C}=\mu+\sigma \sqrt{-\ln (2 \pi)-2 \ln C L R},
$$

where $C L R$ is the cell loss ratio requirement of the traffic. For a group of VCC with different cell loss ratio requirements, there are two approaches to apply the Gaussian approximation formula (3). In the first approach, we treat the aggregate traffic as one traffic stream and adopt the most stringent cell loss ratio requirement. Hence,

$$
\hat{C}=\mu+\sigma \sqrt{-\ln (2 \pi)-2 \ln C L R_{\min }},
$$

where $\mu=\sum_{i=1}^{N} \mu_{i}, \quad \sigma^{2}=\sum_{i=1}^{N} \sigma_{i}^{2}$, and $C L R_{\min }=\min \left(C L R_{1}\right.$, $\left.C L R_{2}, \mathrm{~L}, C L R_{N}\right)$. The approximation formula (4) is refereed to as Approximation 1 in the paper.

In the second approach, the Gaussian formula is applied to calculate the bandwidth requirement of each individual VCC based on its own traffic characteristics and cell loss ratio specification. The total bandwidth required is simply the sum of those required by individual VCC. Hence, we have

$$
\hat{C}=\sum_{i=1}^{N} \hat{C}_{i},
$$

where $\hat{C}_{i}=\mu_{i}+\sigma_{i} \sqrt{-\ln (2 \pi)-2 \ln C L R_{i}}$. Formula

(5) is refereed to as Approximation 2.

In order to investigate how accurate the above Gaussian approximations are, we again use simulations to determine the exact bandwidth required under a particular scheduling scheme. For a given number of VCC with their traffic characteristics and cell loss ratio requirements, we use the bisection search method to find the amount of bandwidth required to satisfy the cell loss ratio requirements of all VCC under each of the four scheduling schemes.

We now examine how well the two Gaussian approximation formulas perform under different cell loss scheduling schemes. First, we consider the case of homogeneous sources which are generated from Class 2 as defined in Table 1. For each of the four cell scheduling schemes, we used the bisection search method with simulations to find the amount of bandwidth required when there are $5,10,15,20,25$, and 30 sources, respectively. For the same set of sources, we calculated the bandwidths corresponding to Approximation 1 and Approximation 2. The resulting bandwidths obtained were so close that we decided to present the results in a bar chart (as shown in Fig. 3) instead of a line graph.

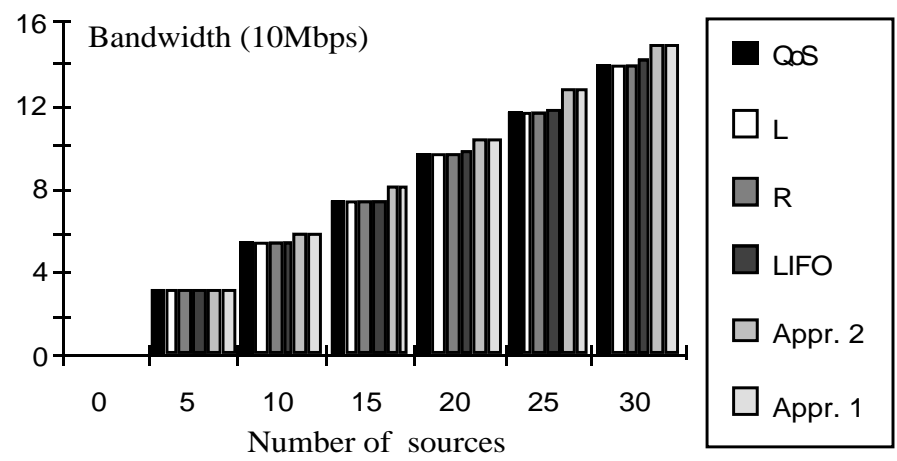

Fig. 3. Comparison of bandwidth requirement: Homogeneous sources

From Fig. 3, we can see that both approximation methods consistently overestimate the bandwidth required by any of the four cell scheduling schemes. Since we are dealing with homogeneous VCC with the same traffic characteristics and the same cell loss ratio requirement, both approximation methods give identical results. In Section 3 we discussed the fact that the Lscheme performs very well on homogeneous sources. Since now we have homogeneous sources with identical QoS requirements, the QoS-scheme and the L-scheme give identical values for bandwidth requirement. Finally the LIFO-scheme performs the worst as it gives, on the average, the highest required bandwidth needed to meet cell loss requirement of all VCC. It is interesting to note that even though LIFO performs the worst, it does not perform drastically different than all of the other schemes in this set of simulation results. We have performed another set of simulation runs using homogeneous sources from Class 1 . These results led to the same conclusions.

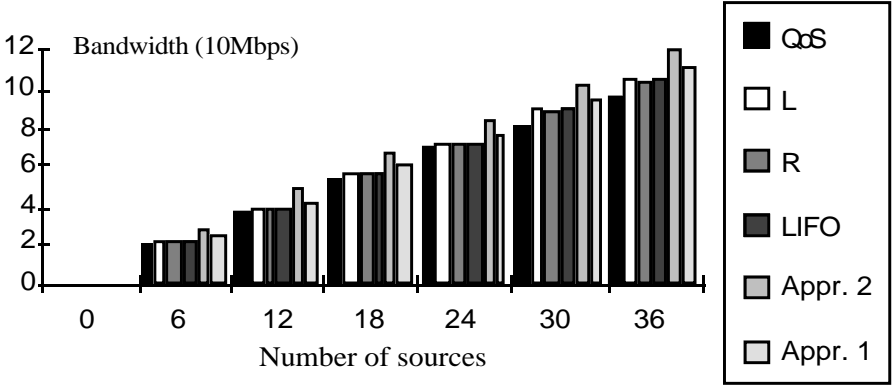

Fig. 4. Comparison of bandwidth requirement: Heterogeneous sources I 
We next consider the case of heterogeneous sources where all three classes defined in Table 1 are used. The first set of runs that we present use sources that are generated from Class 1, Class 2 , and Class 3 in an even proportion. For these runs, we use the following combination of sources from Classes 1,2 , and 3 , respectively: 2-2-2, 4-4-4, 6-6-6, 8-8-8, 10-10-10, and 12-12-12. The bandwidth requirements for each of the cell loss scheduling schemes and for each of Gaussian formulas are given in Fig. 4.

We first observe that the two Gaussian approximation methods again consistently overestimate the bandwidth under each of the cell loss scheduling schemes. However, Approximation 1 seems to perform much better than Approximation 2. To compare the various scheduling schemes, we notice that the required bandwidth when using the QoS-scheme is the lowest among those of other schemes. In fact, as the number of sources is increased the slope of bandwidth curve from the QoS-scheme decreases at a greater rate than that of the other schemes. This observation means that the QoS-scheme performs even better as the number of sources is increased. The power behind the QoS-scheme lies from the fact that this scheme is the only one that tries to satisfy individual QoS requirements from all VCC. It does this by evening out the relative errors that occur when the individual cell loss ratios differ from the corresponding CLR requirement. The $\mathrm{L}$-scheme and the R-scheme perform about the same as the LIFO-scheme.

The next set of runs that we present involves heterogeneous sources generated from Class 1, Class 2 and Class 3 in the proportion $3: 1: 2$. That is, we use the following combination of sources from classes 1,2 , and 3, respectively: 3-1-2, 6-2-4, 9-3-6, 12-4-8, 15-5-10, and 18-6-12. The results are shown in Fig. 5. The observations made from the first set (Fig. 4) extend to this set as well, which gives more credence to our conclusions. We have performed a third set of heterogeneous runs as well with a different proportion of class 1 , class 2 , and class 3 sources. We will not show the results here as the findings are similar to the previous two sets of runs.

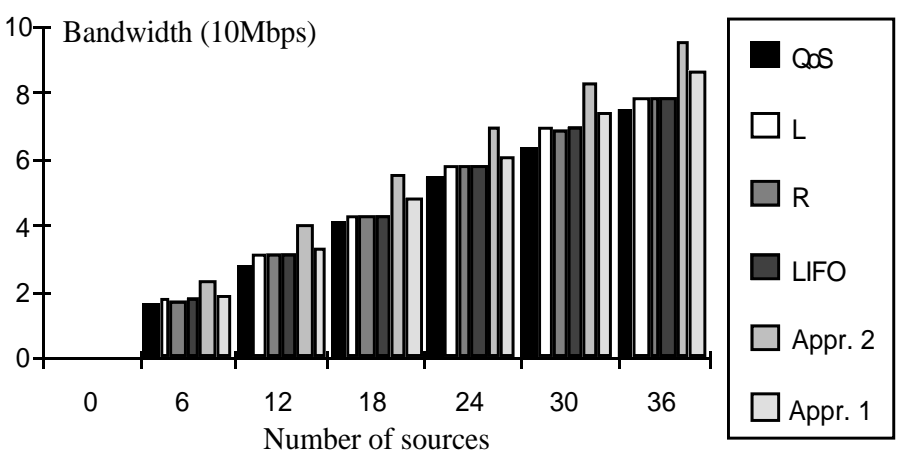

Fig. 5. Comparison of bandwidth requirement: Heterogeneous sources II

We now determine which of the four cell loss scheduling schemes can be coupled with the Gaussian approximations. It is quite clear from the above observations that the two Gaussian approximation methods consistently overestimate the bandwidth required by any of the four cell loss scheduling schemes in both the homogeneous source case and the heterogeneous source case. This implies that if the Gaussian formulas were used to allocate bandwidth to a group of VCC, then no matter which scheduling scheme is used the cell loss ratio requirement of all VCC would be satisfied. Between the two Gaussian approxima- tion formulas, Approximation 1 provides a tighter bound than Approximation 2 and hence it yields a better bandwidth utilization.

Now, the question is: which cell loss scheduling scheme should be adopted? The answer to this question depends on two factors. The first is the tradeoff between bandwidth utilization and the cost of implementation. Clearly, the LIFO-scheme is the most inexpensive one to implement. For the L-scheme, a counter is needed for each VCC and each time the buffer is full a search is needed to find the smallest $L_{i}$ value to decide which cell to discard. Both requirements may be expensive to meet with existing technology. The R-scheme and the QoS-scheme are more expensive to implement. In terms of bandwidth utilization, the QoS-scheme is undoubtedly the most efficient one while the other three schemes perform more or less the same. Therefore, the choice is really between the LIFO-scheme and the QoSscheme. To see how much bandwidth can be saved by using the QoS-scheme instead of the LIFO-scheme, we present the savings in percentage in Table 6 for the two sets of runs shown in Fig. 4 and 5 . Here, we notice that the savings are less than $10 \%$.

Table 6. Savings in bandwidth by the QoS-scheme over the LIFO-scheme

\begin{tabular}{|c|c|c|}
\hline No. of sources & Set 1 (Fig. 4) & Set 2 (Fig. 5) \\
\hline 6 & $4.77 \%$ & $7.13 \%$ \\
\hline 12 & $8.00 \%$ & $9.54 \%$ \\
\hline 18 & $5.30 \%$ & $4.68 \%$ \\
\hline 24 & $3.12 \%$ & $6.41 \%$ \\
\hline 30 & $8.01 \%$ & $8.92 \%$ \\
\hline 36 & $8.37 \%$ & $4.88 \%$ \\
\hline
\end{tabular}

The second factor is the existence of a coupling bandwidth allocation algorithm that makes use of the specific features of the chosen scheme to improve bandwidth utilization. Due to complexity of the QoS-scheme, there does not seem to be any simple bandwidth allocation algorithm that is particularly suitable for the QoS-scheme. If the Gaussian approximation formula (4) is used to allocate bandwidth, then there is really no savings in bandwidth when the QoS-scheme rather than the LIFO-scheme is used.

Table 7. Bandwidth over-allocation by Gaussian Approximation

\begin{tabular}{|c|c|c|}
\hline No. of sources & Set 1 (Fig. 4) & Set 2 (Fig. 5) \\
\hline 6 & $10.03 \%$ & $10.31 \%$ \\
\hline 12 & $6.06 \%$ & $4.84 \%$ \\
\hline 18 & $7.68 \%$ & $10.61 \%$ \\
\hline 24 & $6.04 \%$ & $4.25 \%$ \\
\hline 30 & $4.40 \%$ & $6.03 \%$ \\
\hline 36 & $4.12 \%$ & $8.48 \%$ \\
\hline
\end{tabular}

From the above discussions, we can conclude that a practically implementable solution is to adopt the simple LIFO-scheme coupled with the bandwidth allocation algorithm based on the Gaussian approximation formula given in (4). To examine the accuracy of the Gaussian approximation more closely, we present in Table 7 the percentage of over-estimation of bandwidth by (4) as compared to the exact bandwidth requirement determined by the simulations for the LIFO-scheme. From this table, we observe that the over-estimation is in most cases well under $10 \%$ and is only slightly over $10 \%$ in some cases. 


\section{Conclusions}

In this paper, we studied the problems of cell loss scheduling and bandwidth allocation for an ATM multiplexer loaded with several VCC carrying heterogeneous VBR video conferencing traffic. We introduced a new cell loss scheduling scheme (called the QoS-scheme) that intends to eliminate the effect of traffic interference among different VCC and at the same time guarantee their individual QoS requirements. We compared the performance of the QoS-scheme with those of other scheduling schemes, including the LIFO-scheme, using detailed simulations of the traffic model. We found that the QoS-scheme requires the smallest amount of bandwidth, as compared to the other schemes, needed to guarantee different individual cell loss requirements of the VCC involved. In theory, we conjecture that the QoS-scheme is the most efficient scheme in terms of bandwidth utilization. However, it may be too expensive to be implemented in a switch with today's technology. The major contribution of the QoSscheme in this paper is its role as an ideal model with which we can judge the performance of the existing scheduling schemes and bandwidth allocation algorithms and determine a suitable coupling between the two. From our comparison study, we found that the savings in bandwidth that can be achieved by using the QoS-scheme instead of the simple LIFO-scheme is only less than $10 \%$ in all cases considered in this study. This suggests that the LIFO-scheme is not far off the ideal situation.

For bandwidth allocation, we extended the Gaussian approximation method to the traffic model considered in this paper. We derived two formulas for the calculation of bandwidth requirement. In the first one, the bandwidth requirement is calculated based on the traffic characteristics of the aggregate traffic and the most stringent QoS requirement. In the second method, it is calculated as the sum of the bandwidth requirements of individual VCC, each being computed based on individual traffic characteristics and QoS requirement. Our studies showed that both methods consistently over-estimate the bandwidth required under any of the four scheduling schemes. Furthermore, the first method gives a tighter upper bound on bandwidth requirement and therefore it is more efficient in terms of bandwidth utilization. When this method is applied to the LIFO scheduling scheme, the over-estimation in bandwidth is in most cases under $10 \%$.

In summary, our findings seem to suggest that the LIFOscheme is still the most attractive cell loss scheduling scheme: It is inexpensive and uses less than $10 \%$ extra bandwidth as compared with the most efficient scheme. When the Gaussian approximation (method 1) is coupled with the LIFO-scheme, about $10 \%$ more bandwidth will be required. In other words, if the LIFO-scheme is adopted, developing a more efficient bandwidth allocation algorithm can at most improve bandwidth utilization by about $10 \%$. On the other hand, if the sophisticated QoS-scheme is implemented, then a new allocation algorithm can improve bandwidth utilization by a factor of roughly $20 \%$.

\section{Acknowledgment}

The authors were grateful to Dr. Daniel P. Heyman of Bellcore for providing the video conferencing data which are critical to many of the findings in this work and to Dr. Jie Pan for many helpful suggestions.
[1] J. Bae and T. Suda, Survey of traffic control schemes and protocols in ATM networks, Proc. of IEEE, 79(2) (1991), pp. 170-189.

[2] H. Chu, D. Tsang, and T. Yang, Call admission control of Teleconferencing VBR video traffic in ATM networks, to appear in IEEE ICC'95.

[3] C. Cooper and K. Park, Toward a Broadband Congestion Control Strategy, IEEE Network, (May 1990), pp. 18-23.

[4] A. Elwalid and D. Mitra, Effective Bandwidth of General Markovian Traffic Sources and Admission Control of High Speed Networks, IEEE/ACM Trans. on Networking, vol. 1, pp. 329-343, June 1993.

[5] R. Gibbens and P. Hunt, Effective Bandwidth for MultiType UAS Channels, Queueing Systems, vol. 9, pp. 17-28, 1991. [6] H. Gilbert, O. Aboul-Magd and V. Phung, Developing a Cohesive Traffic Management Strategy for ATM Networks, IEEE Communication Magazine, (October 1991), pp. 36-45.

[7] Guerin, R., Ahmadi, H., and Naghshineh, M., Equivalent Capacity and its Application to Bandwidth Allocation in HighSpeed Networks, IEEE Journal on Selected Areas in Communication, vol. 9, no. 7, pp. 968-981, Sept., 1991.

[8] I. Habib and T. Saadawi, Controlling Flow and avoiding Congestion in Broadband Networks, IEEE Communication Magazine, (October 1991), pp. 46-53.

[9] Heyman, D.P., Tabatabai, A. and Lakshman, T.V., Statistical Analysis and Simulation Study of Video Teleconference Traffic in ATM Networks, IEEE Trans. on Circuits and Systems for Video Technology, vol. 2, no. 1, pp. 4959, Mar., 1992.

[10] D. Hong and T. Suda, Congestion Control and Prevention in ATM networks, IEEE Network, (July 1991), pp. 10-16.

[11] J. Hui, Resource Allocation for Broadband Networks, IEEE JSAC, 6(9) (1988), pp. 1598-1608.

[12] R. Jain, Congestion Congestion in Computer Networks: Issues and Trends, IEEE Network, (May 1990), pp. 24-30.

[13] G. Keisidis, J. Walrand, and C. Chang, Effective Bandwidth for Multiclass Markov Fluids and other ATM sources, IEEE/ACM Trans. on Networking, vol. 1, pp. 424-428, August 1993.

[14] A. Lazar and G. Pacifici, Control of Resources in Broadband Networks with Quality of Service Guarantees, IEEE Communication Magazine, (October 1991), pp. 66-73.

[15] J. Le Boudec, The Asynchronous Transfer Mode: A Tutorial, Computer Networks and ISDN Systems, 24 (1992), pp. 279-309.

[16] Y. Miyao, Bandwidth Allocation in ATM Networks that Guarantee Multiple QoS Requirements ICC'93.

[17] T. Murase, H. Suzuki and T. Takeuchi, A Call Admission Control for ATM Networks based on Individual Multiplexed Traffic Characteristics, Proc. ICC'91, pp. 193-198, 1991.

[18] C. O'Neil, Comparison of ATM Network Congestion Control Methods, A.T.R., 26(2) (1992), pp. 31-38.

[19] R. Onvural, Asynchronous Transfer Mode Networks: Performance Issues, Artech House, London, 1994.

[20] T. Yang and H. Li, Individual Cell Loss Probabilities and Traffic Interference in ATM Networks, ICC'93, Geneva, Switzerland, May 1993.

[21] T. Yang, D. Tsang, and S. Li, Cell scheduling and bandwidth allocation for a class of VBR video connections, IEEE Workshop on Visual Signal Processing and Communications, New Brunswick, New Jersey, September 1994.

\section{References}

